\title{
Mediennutzung und -wahrnehmung von Bundestagsabgeordneten: Ersetzen oder ergänzen Online-Medien klassische Informations- und Kommunikationskanäle?
}

\author{
Marco Dohle und Uli Bernhard*
}

Ob im Plenum oder Ausschuss: Parlamentarier haben ihr Smartphone fast immer in der Hand oder zumindest im Blick; und so gut wie jeder hat einen Online-Auftritt, auf dem er seine politischen Ziele, Tätigkeiten und Erfolge verkündet und nicht zuletzt über sich als Privatperson informiert. ${ }^{1}$

Das rasante Wachstum des Onlinegebrauchs wirft die Frage auf, wie dominant OnlineMedien mittlerweile für die politische Information und Kommunikation von Abgeordneten geworden sind: Haben sie klassische Formen der Mediennutzung weitgehend verdrängt? Oder haben die Abgeordneten ihr Informations- und Kommunikationsrepertoire um Online-Medien erweitert, ohne dabei die Bedeutung von Zeitungen, Fernsehen oder auch der direkten Kontakte mit Journalisten und Bürgern gering zu schätzen? Und wie werden Online- im Vergleich zu Offline-Medien eingeschätzt, etwa was die Eignung zur politischen Information, die Reichweite oder die politische Wirkkraft angeht? Diese Fragen sollen anhand der Resultate von zwei Befragungen der Abgeordneten des 17. Deutschen Bundestags beantwortet werden.

Die Daten geben Hinweise darauf, wie politische Kommunikation in einer sich wandelnden Medienwelt stattfindet: Welche Wege der Kommunikation nutzen die Parlamentarier selbst, und auf welchem Wege sind sie erreichbar? Abgeordnete gelten als gesellschaftliche Elite. Es ist daher zu klären, ob sie sich in ihrer Mediennutzung und -wahrnehmung von der Gesamtbevölkerung unterscheiden - etwa dadurch, dass sie Wandlungsprozesse schneller vollziehen und gesellschaftlich bedeutsame Medienumbrüche vorantreiben. Ebenso denkbar ist jedoch, dass gerade Politiker Veränderungen im Mediennutzungsverhalten der Bevölkerung wahrnehmen und sich (vielleicht voreilig) daran orientieren. Darüber hinaus ermöglichen Online-Medien zwar einerseits neue Formen und Wege der Information und Kommunikation. Andererseits bedeutet ihre Integration in ein bestehendes Mediennutzungs- und Kommunikationsrepertoire zusätzlichen Aufwand. Fraglich ist, wie die Abgeordneten, deren Zeitbudget ohnehin sehr begrenzt ist, mit diesen Herausforderungen umgehen.

* Diese Publikation entstand im Rahmen der von der Deutschen Forschungsgemeinschaft (DFG) geförderten Forschergruppe „Politische Kommunikation in der Online-Welt“ (1381), Teilprojekt 3. Wir danken allen Abgeordneten des Deutschen Bundestags herzlich für ihre Teilnahmebereitschaft an den Befragungsstudien, auf denen dieser Beitrag basiert.

1 Wenn in diesem Beitrag aus Gründen der Lesbarkeit für die Bezeichnung von Personengruppen die grammatikalisch maskulinen Formen genutzt werden, sind damit immer Männer und Frauen gemeint. 


\section{Stand der Forschung: Abgeordnete und ihr Gebrauch von (Online-)Medien}

Zahlreiche Studien haben sich mittlerweile mit den Onlineaktivitäten von Parlamentariern befasst. ${ }^{2}$ Es handelt sich teilweise um Analysen von Inhalten oder Nutzungsfrequenzen, teilweise um Befragungen. Die Untersuchungen betreffen zum Teil die Abgeordneten des Deutschen Bundestags, aber auch anderer Parlamente; sie konzentrieren sich auf spezifische Zeiträume wie etwa Wahlkampfphasen oder auf den politischen Alltag wie etwa die Wahlkreisarbeit. Zum Teil werden zudem politische Ebenen oder Nationen verglichen. Studien mit einem Fokus auf Bundestagsabgeordnete ${ }^{3}$ zeigen dabei unter anderem, dass nahezu alle MdBs über eine eigene Website verfügen. Weit über die Hälfte ist zudem aktiver SocialMedia-Nutzer - und besitzt beispielsweise ein eigenes Facebook-Profil oder verbreitet mehr oder weniger intensiv Mitteilungen per Twitter. Das Ausmaß an Onlineaktivitäten der Bundestagsabgeordneten ist also enorm. Die Gründe für dieses Engagement sind vielfältig - sie reichen vom Wunsch, auf politische Erfolge hinzuweisen, bis zu dem Ziel, die Aufmerksamkeit auf wichtige Themen zu lenken. Ebenso vielfältig sind die Zielgruppen: vom eigenen Netzwerk bis hin zu allen Wählern.

Online-Medien können von Bundestagsabgeordneten außerdem als Quelle politischer Informationen genutzt werden. Auch hierzu liegen Daten vor, wenn auch in geringerem Maße als zur aktiven Online-Kommunikation. Die Ergebnisse einer im Jahr 2012 durchgeführten Befragungsstudie ${ }^{4}$ legen dabei nahe, dass MdBs sehr intensive Internetnutzer sind. Die Informationssuche und der Wunsch, auf dem Laufenden zu bleiben, wurden als bevorzugte Nutzungsgründe genannt.

2 Zum Beispiel für deutsche Abgeordnete: Annette Knaut, Politikvermittlung online. Abgeordnete des Deutschen Bundestages im Web 2.0, in: Gerhard Göhler / dies. / Cornelia Schmalz-Jacobsen / Christian Walther (Hrsg.), Medien / Demokratie. Politik und Journalismus in Berlin, Frankfurt am Main 2010, S. 9 - 36; Miriam Meckel / Christian P. Hoffmann / Eliane Bucher / Anne Suphan, Politiker im Netz. Der Social Media Activity Index 2011 des 17. Deutschen Bundestags. Zwischenbericht, St. Gallen 2011; Miriam Meckel / Christian P. Hoffmann / Anne Suphan / Robin Poëll, Politiker im Netz. Treiber und Hürden der Social Media-Nutzung unter Bundes- und Landtagsabgeordneten. Abschlussbericht, St. Gallen 2013; Sven T. Siefken, Repräsentation vor Ort: Selbstverständnis und Verhalten von Bundestagsabgeordneten bei der Wahlkreisarbeit, in: ZParl, 44. Jg. (2013), H. 3, S. 486 - 506; Jens Tenscher, MPs and the Internet - An Empirically Based Typology, in: The Journal of Legislative Studies, 20. Jg. (2014), H. 3, S. 305 - 320; ders. I Laura Will, Abgeordnete online? Internetaktivitäten und -bewertungen der Mitglieder des Deutschen Bundestages, in: ZParl, 41. Jg. (2010), H. 3, S. 604 - 618; Caja Thimm / Jessica Einspänner / Mark Dang-Anh, Twitter als Wahlkampfmedium. Modellierung und Analyse politischer Social-Media-Nutzung, in: Publizistik, 57. Jg. (2012), H. 3, S. 293 - 313; Jens Wolling / Anja Schmolinsky / Martin Emmer, Politiker vernetzt: Wie und warum sich Landtagsabgeordnete online präsentieren, in: Jens Wolling / Markus Seifert / Martin Emmer (Hrsg.), Politik 2.0? Die Wirkung computervermittelter Kommunikation auf den politischen Prozess, Baden-Baden 2010, S. 59 - 83; Thomas Zittel, Entmedialisierung durch Neue Medien? Direkte Wählerkommunikation im WWW aus der Sicht von Abgeordneten des Deutschen Bundestages, in: Frank Marcinkowski / Barbara Pfetsch (Hrsg.), Politik in der Mediendemokratie, PVS-Sonderheft 42, Wiesbaden 2009, S. $367-389$.

3 Zum Beispiel Miriam Meckel / Christian P. Hoffmann / Eliane Bucher / Anne Suphan, a.a.O. (Fn. 2); Jens Tenscher / Laura Will, a.a.O. (Fn. 2). Siehe auch bereits Daniel Pontzen, Nur Bild, BamS und Glotze? Medialisierung der Politik aus Sicht der Akteure, Münster 2006.

4 Vgl. Miriam Meckel / Christian P. Hoffmann / Anne Suphan / Robin Poëll, a.a.O. (Fn. 2). 
Detaillierte Analysen verdeutlichen, dass es Unterschiede zwischen den Abgeordneten gibt. Vor allem das Alter scheint eine gewichtige Rolle zu spielen: Je jünger die Abgeordneten sind, desto intensiver verwenden sie Online-Medien.5 Auch differieren die Einschätzungen, wie nützlich diese für politische Zwecke sind: Je höher dabei der erwartete Nutzen, desto intensiver der Onlinegebrauch. ${ }^{6}$

Wie aber verhält sich starker Gebrauch von Online-Angeboten zu der Nutzung traditioneller Medien? Insbesondere Zeitungen (nicht nur die überregionalen, sondern vor allem die Lokal-/Regionalpresse) haben älteren Studien zufolge unter Bundestagsabgeordneten eine hervorgehobene Bedeutung zur politischen Information und zur Verbreitung ihrer eigenen Botschaften, mehr noch als das Fernsehen. ${ }^{7}$ Aktuelle Daten zur Mediennutzung speziell von MdBs sind rar. Eine Ausnahme stellt eine Untersuchung von Bundestags- und Landtagsabgeordneten aus dem Jahr 2005 dar: Den dortigen Angaben zufolge nutzten die befragten Abgeordneten Fernsehen, Zeitungen und Internet etwa gleichermaßen stark konkret jeweils knapp über eine Stunde täglich. ${ }^{8}$

Dagegen liegen weitaus mehr neuere Studien vor, in denen Parlamentarier um Auskunft darüber gebeten wurden, wie sie die Relevanz von Medien im politischen Kontext einschätzen. ${ }^{9}$ Durchgängig nehmen demnach Abgeordnete einen außerordentlich starken Einfluss von Medien auf die Bevölkerung, aber auch auf politische Prozesse wahr. Eine entsprechend große Notwendigkeit schreiben sie ihren eigenen Medien- und Kommunikationsaktivitäten zu. Falls in diesen Befragungen zwischen verschiedenen Medien differenziert wurde, wird den Zeitungen und dem Fernsehen stets eine höhere politische Bedeutung zugewiesen als dem Internet - zum Beispiel hinsichtlich des wahrgenommenen Einflusses

5 Zum Beispiel Miriam Meckel / Christian P. Hoffmann / Eliane Bucher / Anne Suphan, a.a.O. (Fn. 2); Jens Tenscher, a.a.O. (Fn. 2); ders. / Laura Will, a.a.O. (Fn. 2). Allerdings zeigt sich dieser Befund nicht einhellig. Die Entscheidung für eine eigene Website stand laut einer Befragung von Landtagsabgeordneten beispielsweise in keinem Zusammenhang mit dem Alter der Parlamentarier: Jens Wolling / Anja Schmolinsky / Martin Emmer, a.a.O. (Fn. 2).

6 Zum Beispiel Jens Wolling / Anja Schmolinsky / Martin Emmer, a.a.O. (Fn. 2).

7 Zum Beispiel Dietrich Herzog / Hilke Rebenstorf / Camilla Werner / Bernhard Weßels, Abgeordnete und Bürger. Ergebnisse einer Befragung der Mitglieder des 11. Deutschen Bundestages und der Bevölkerung, Opladen 1990; Werner J. Patzelt, Abgeordnete und Journalisten, in: Publizistik, 36. Jg. (1991), H. 3, S. 315 - 329; ders., Abgeordnete und ihr Beruf. Interviews - Umfragen - Analysen, Berlin 1995; Henry Puhe / H. Gerd Würzberg, Lust und Frust. Das Informationsverhalten des deutschen Abgeordneten. Eine Untersuchung, Köln 1989.

8 Vgl. Daniel Pontzen, a.a.O. (Fn. 3), S. 141.

9 Vgl. Marco Doble / Christoph Blank / Gerhard Vowe, Wie sehen Parlamentarier den Einfluss der Medien? Ergebnisse einer Befragung der Bundestagsabgeordneten, in: ZParl, 43. Jg. (2012), H. 2, 376 - 388; Hans Mathias Kepplinger, Rivalen um Macht und Moral: Bundestagsabgeordnete und Hauptstadtjournalisten, in: Hanna Kaspar / Harald Schoen / Siegfried Schumann / Jürgen R. Winkler (Hrsg.), Politik - Wissenschaft - Medien, Wiesbaden 2009, S. 307 - 321; Klaus-Dieter Müller, www.Internet-Abgeordnete.de: Die digitale Welt und das Rollenselbstverständnis von Abgeordneten, Berlin 2007; Daniel Pontzen, a.a.O. (Fn. 3); Jens Tenscher, Salto mediale? Medialisierung aus der Perspektive deutscher Landtagsabgeordneter, in: Michael Edinger / Werner J. Patzelt (Hrsg.), Politik als Beruf, PVS-Sonderheft 44, Wiesbaden 2010, S. 375 - 395; diese Ergebnisse zeigen sich auch mit Blick auf andere Länder, zum Beispiel für Schweden oder Österreich: Jesper Strömbäck, Mediatization and Perceptions of the Media's Political Influence, in: Journalism Studies, 12. Jg. (2011), H. 4, S. 423 - 439; Jens Tenscher, Nur ZiB und Krone? Medienorientierungen österreichischer Abgeordneter, in: SWS-Rundschau, 52. Jg. (2012), H. 3, S. $321-342$. 
auf die Bürger. Gerade die Präsenz in den Printmedien wird, so das Ergebnis einer Studie unter Landtagsabgeordneten, zudem als ein entscheidender Teil der eigenen Politikvermittlung eingeschätzt; als wichtiger gelten den Parlamentariern lediglich die persönlichen Gespräche mit Bürgern. ${ }^{10}$ Allerdings wird von Abgeordneten auch ein Bedeutungszuwachs des Internets prognostiziert. ${ }^{11}$

Aktuelle, vergleichende Daten zur Nutzung von Online- und traditionellen Medien wie den Zeitungen oder dem Fernsehen fehlen jedoch weitgehend. Angesichts der fortgeschrittenen Ausdifferenzierung der Online-Kommunikation erscheint es sinnvoll zu prüfen, ob beispielsweise der hohe Stellenwert der traditionellen Medien für die Abgeordneten noch Bestand hat. Zudem existieren keine neueren Befunde dazu, für wie geeignet sie verschiedene Online- und Offline-Medien halten, wenn es darum geht, sich über Politik zu informieren. Auch fehlen Daten, welche Einschätzungen die Abgeordneten zur politischen Nutzung verschiedener Medien durch die Bevölkerung haben. Und schließlich wurde bislang zwar erhoben, für wie bedeutsam die Parlamentarier Medien einschätzen, selten aber konkret, welchen politischen Einfluss sie ihnen auf die Bevölkerung unterstellen. Solche subjektiven Wahrnehmungen über Eignung, Nutzung und Wirkungen sind relevant, da sie individuelle Konsequenzen haben können, etwa für den eigenen Mediengebrauch oder für die Planung und Durchführung von Kommunikationsaktivitäten. ${ }^{12}$

Grundsätzlich stellt sich also die Frage, ob Online-Medien die klassischen Informationsund Kommunikationskanäle ersetzen oder ob sie als Ergänzung dieser Kanäle genutzt und wahrgenommen werden. Daraus lassen sich vier konkrete Fragen ableiten:

(1) Wie intensiv nutzen die Abgeordneten des Bundestags Online- und Offline-Medien, um sich über Politik zu informieren? (2) Wie intensiv nutzen sie Online- und Offline-Kanäle, um aktiv über ihre politische Arbeit zu kommunizieren? (3) Wie nehmen die MdBs verschiedene Online- und Offline-Medien wahr, und zwar im Hinblick auf die Eignung zur politischen Information, auf die Reichweite und auf den Einfluss auf die Bevölkerung? (4) Wie hängt der tatsächliche Mediengebrauch der Abgeordneten mit ihren Wahrnehmungen zusammen?

$\mathrm{Da}$ in der vorliegenden Untersuchung zu zwei Erhebungszeiträumen Daten erhoben wurden, ist diesbezüglich auch ein zeitlicher Vergleich möglich, der - in begrenztem Umfang - Aufschluss über mögliche Wandlungsprozesse erlaubt.

\section{Methode}

Die Forschungsfragen werden mit Daten von zwei standardisierten Befragungen beantwortet. Im Frühjahr 2012 und im Frühjahr 2013 wurden alle 620 Mitglieder des 17. Deutschen Bundestages jeweils per Post angeschrieben und gebeten, an der Befragung teilzuneh-

10 Vgl. Jens Tenscher, a.a.O. (Fn. 9), S. 390.

11 Vgl. Daniel Pontzen, a.a.O. (Fn. 3).

12 Zum Beispiel Jonathan Cohen / Yariv Tsfati / Tamir Sheafer, The Influence of Presumed Media Influence in Politics. Do Politicians' Perceptions of Media Power Matter?, in: Public Opinion Quarterly, 72. Jg. (2008), H. 2, S. 331 - 344; Dorothee Marx, Landtagsabgeordnete im Fokus der Medien. Ihre Sicht auf Entstehung, Wirkung und Qualität landespolitischer Berichterstattung, Baden-Baden 2009; im Überblick: Marco Dohle, Third-Person-Effekt, Baden-Baden 2013. 


\begin{tabular}{|c|c|c|c|}
\hline & Stichprobe 2012 & Stichprobe 2013 & Bundestag gesamt \\
\hline \multicolumn{4}{|l|}{ Geschlecht } \\
\hline weiblich & 28,1 & 29,5 & 32,9 \\
\hline männlich & 71,9 & 70,5 & 67,1 \\
\hline \multicolumn{4}{|l|}{ Geburtsjahr } \\
\hline 1950 oder früher & 25,8 & 23,8 & 21,3 \\
\hline 1951 bis 1960 & 29,0 & 33,8 & 34,0 \\
\hline 1961 bis 1970 & 29,0 & 25,4 & 27,6 \\
\hline 1971 bis 1980 & 14,8 & 15,4 & 15,2 \\
\hline 1981 oder später & 1,2 & 1,5 & 1,9 \\
\hline \multicolumn{4}{|l|}{ Fraktionszugehörigkeit } \\
\hline $\mathrm{CDU} / \mathrm{CSU}$ & 38,6 & 29,2 & 38,2 \\
\hline SPD & 23,9 & 30,1 & 23,6 \\
\hline FDP & 17,4 & 18,1 & 15,0 \\
\hline Bündnis 90/Die Grünen & 7,0 & 6,9 & 11,0 \\
\hline Die Linke & 13,0 & 15,3 & 12,3 \\
\hline
\end{tabular}

men. ${ }^{13}$ Jeweils zwei und sechs Wochen nach dem Erstkontakt wurden die Parlamentarier per E-Mail an die Teilnahme erinnert. Diese Mails enthielten einen Link zu einem OnlineFragebogen, der identisch mit dem postalisch verschickten Fragebogen war.

An der Erhebung im Jahr 2012 haben sich 194 Abgeordnete beteiligt (Ausschöpfungsquote: 31,3 Prozent); 2013 nahmen $149 \mathrm{MdBs}$ an der Befragung teil (Ausschöpfungsquote: 24,0 Prozent) ${ }^{14}$ Beide Stichproben sind hinsichtlich zentraler Variablen kaum verzerrt gegenüber der Grundgesamtheit. 2013 nahmen allerdings überproportional viele SPD-Abgeordnete teil, während Unions-Abgeordnete unterrepräsentiert sind (vgl. Tabelle 1).

Der Fragebogen umfasste in beiden Jahren identische Variablen, so dass ein Vergleich der Befunde möglich ist. Zum einen wurden die Parlamentarier gefragt, wie häufig sie sich aus Online- und Offline-Medien über Politik informieren und wie intensiv sie das Internet allgemein nutzen. ${ }^{15}$ Zum anderen sollten sie Angaben zu ihren Online- und Offline-Kom-

13 Die zweite Befragungswelle wurde aus zwei Gründen im Frühjahr 2013 durchgeführt: Einerseits sollte der zeitliche Abstand zur ersten Welle möglichst groß sein, um etwaige Entwicklungen erfassen zu können. Andererseits sollten auch in der zweiten Welle die Mitglieder des 17. Deutschen Bundestags befragt werden, und dies außerhalb der heißen Wahlkampfphase.

14 Die Ausschöpfungsquote ähnelt damit den Quoten vergleichbarer Befragungen von Bundestagsabgeordneten - siehe zum Beispiel Bernhard Weßels, Abgeordnetenbefragung 2003. Kurzfassung und Dokumentation der Ergebnisse, Berlin 2003; http://www.wzb.eu/sites/default/files/zkd/ dsl/ber-fin1-all1.pdf (Abruf am 12. März 2014); Hans Mathias Kepplinger, a.a.O. (Fn. 9); Jens Tenscher / Laura Will, a.a.O. (Fn. 2); Marco Doble / Christoph Blank / Gerhard Vowe, a.a.O. (Fn. 9).

15 Itemformulierungen: „Wie häufig nutzen Sie die folgenden Medien, um sich über politische Themen zu informieren?" / „Und wie lange, grob gerechnet, nutzen Sie an einem durchschnittlichen Tag das Internet?" 
munikationsaktivitäten machen. ${ }^{16}$ In diesem Zusammenhang wurde auch gefragt, ob sie über eine eigene Website verfügen und wie intensiv sie diese pflegen. ${ }^{17}$ Darüber hinaus sollten die Befragten angeben, für wie geeignet sie verschiedene Online- und Offlinemedien zur politischen Kommunikation halten ${ }^{18}$, welche Reichweite sie ihnen zuschreiben ${ }^{19}$ und wie stark sie deren politischen Einfluss auf die Bevölkerung einschätzen. ${ }^{20}$

\section{Ergebnisse}

\subsection{Informationsaktivitäten der Bundestagsabgeordneten}

Die Befunde zeigen, dass Zeitungen intensiver genutzt werden als das Fernsehen und nach wie vor auch intensiver als Online-Medien (vgl. Tabelle 2): Nahezu alle Befragten informierten sich in den beiden Jahren der Studie mindestens mehrmals in der Woche aus der Presse über Politik, die meisten davon täglich. Rund 87 Prozent schalteten täglich oder mehrmals in der Woche das Fernsehen ein, um sich über das politische Geschehen zu informieren. Ebenfalls stark ausgeprägt ist die Nutzung von Online-Nachrichtenseiten: Sie wurden in beiden Jahren von rund 90 Prozent der Abgeordneten täglich oder mehrfach in der Woche besucht.

\begin{tabular}{|c|c|c|c|c|c|c|c|c|c|c|}
\hline & \multicolumn{2}{|c|}{ Täglich } & \multicolumn{2}{|c|}{$\begin{array}{c}\text { Mehrmals } \\
\text { in der Woche }\end{array}$} & \multicolumn{2}{|c|}{$\begin{array}{l}\text { Mehrmals } \\
\text { im Monat }\end{array}$} & \multicolumn{2}{|c|}{ Seltener } & \multicolumn{2}{|c|}{$\mathrm{Nie}$} \\
\hline & 2012 & 2013 & 2012 & 2013 & 2012 & 2013 & 2012 & 2013 & 2012 & 2013 \\
\hline Fernsehen & 61,3 & 60,4 & 25,3 & 26,8 & 4,1 & 5,4 & 7,7 & 6,7 & 1,5 & 0,7 \\
\hline Zeitungen & 88,5 & 95,3 & 9,9 & 4,7 & 1,6 & 0,0 & 0,0 & 0,0 & 0,0 & 0,0 \\
\hline $\begin{array}{l}\text { Nachrichtenseiten im } \\
\text { Internet }\end{array}$ & 69,3 & 66,4 & 23,4 & 23,5 & 3,6 & 5,4 & 3,1 & 4,7 & 0,5 & 0,0 \\
\hline $\begin{array}{l}\text { Facebook oder andere } \\
\text { Soziale Netzwerke }\end{array}$ & 33,0 & 42,6 & 19,1 & 14,9 & 7,2 & 10,1 & 19,6 & 14,9 & 21,1 & 17,6 \\
\hline Twitter & 19,0 & 19,7 & 7,9 & 8,2 & 6,3 & 5,4 & 13,8 & 18,4 & 52,9 & 48,3 \\
\hline Weblogs & 5,3 & 1,4 & 9,0 & 12,9 & 19,7 & 14,3 & 35,1 & 35,4 & 30,9 & 36,1 \\
\hline YouTube & 1,6 & 0,0 & 11,1 & 7,5 & 17,5 & 17,1 & 38,1 & 48,6 & 31,7 & 26,7 \\
\hline $\begin{array}{l}\text { Internetauftritte der } \\
\text { Parteien }\end{array}$ & 5,2 & 3,4 & 18,7 & 20,9 & 34,2 & 36,5 & 37,3 & 35,1 & 4,7 & 4,1 \\
\hline \multicolumn{11}{|c|}{$\begin{array}{l}\text { Anmerkung: Stichprobe 2012: } \mathrm{n}=188-194 ; \text { Stichprobe 2013: } \mathrm{n}=146-149 \text {. } \\
\text { Lesebeispiel: } 61,3 \text { Prozent der Befragten nutzten im Jahr } 2012 \text { täglich das Fernsehen zur politischen } \\
\text { Information. } \\
\text { Quelle: Eigene Erhebung. }\end{array}$} \\
\hline
\end{tabular}

16 Itemformulierungen: „Wie stark nutzen Sie oder Ihre Mitarbeiter die folgenden Mittel, um über Ihre alltägliche politische Arbeit zu informieren?"

17 Itemformulierungen: „Sind Sie gegenwärtig mit einer eigenen Website im Internet präsent?“ / „Wie viele Stunden investieren Sie und/oder Ihre Mitarbeiterinnen beziehungsweise Mitarbeiter durchschnittlich in der Woche für die Pflege Ihrer Website?“

18 Itemformulierung: „Für wie geeignet halten Sie die folgenden Medien und Internetangebote, wenn man sich über aktuelle politische Ereignisse informieren will?"

19 Itemformulierung: „Was glauben Sie: Wie viele Menschen nutzen in Deutschland die folgenden Medien, um sich über Politik zu informieren?"

20 Itemformulierung: „Wenn Sie an [...] denken: Wie stark ist Ihrer Meinung nach dessen politischer Einfluss auf die Bevölkerung in Deutschland allgemein?" 
Die in der Untersuchung abgefragten spezifischen Online-Angebote wurden dagegen weitaus weniger zur politischen Information genutzt: Etwas mehr als die Hälfte der Abgeordneten informierte sich täglich oder mehrmals pro Woche via Facebook, etwas mehr als ein Viertel per Twitter. Ein größerer Teil der Befragten nahm diese Social-Media-Angebote gar nicht an. Noch seltener suchten die Abgeordneten Weblogs und YouTube auf, um sich über Politik zu informieren. Auch die Internetauftritte der Parteien wurden 2012 und 2013 nur von rund einem Viertel der Befragten täglich oder mehrmals in der Woche zu diesem Zweck besucht. Ganz allgemein nutzten die Abgeordneten das Internet im Durchschnitt rund drei Stunden am Tag (2012: 3,08 Stunden/Tag, Standardabweichung: 2,47; 2013: 2,94 Stunden/Tag, Standardabweichung: 1,87).

Ein Vergleich der beiden Jahre offenbart ein weitgehend stabiles Nutzungsverhalten. Auffällig ist jedoch, dass Facebook oder andere Soziale Netzwerke 2013 von deutlich mehr MdBs (42,6 Prozent) täglich zur politischen Information besucht wurden als noch 2012 (33,0 Prozent).

\subsection{Kommunikationsaktivitäten der Bundestagsabgeordneten}

Auch im Hinblick auf das aktive Kommunikationsverhalten der Abgeordneten zeigt sich, dass Online-Kanäle herkömmliche Wege der Kommunikation nicht ersetzt haben, sondern ergänzen (vgl. Tabelle 3).

Zwar ist unter den in den Befragungen berücksichtigten Kommunikationsaktivitäten jeweils die eigene Website der am intensivsten genutzte Kanal. Im Jahr 2013 unterhielten 98,0 Prozent der Befragten einen solchen Webauftritt (2012: 96,9 Prozent), der mit einem durchschnittlichen Aufwand von 3,8 Stunden pro Woche gepflegt wurde (2012: 4,9 Stunden/Woche). Allerdings verbreiteten die Parlamentarier ihre Positionen daneben auch sehr intensiv mittels Presseerklärungen und Gesprächen mit Journalisten sowie in Bürgersprechstunden. Wenn die Befragten über Online-Kanäle kommunizierten, geschah dies - abgesehen von der eigenen Website - am ehesten über Chats oder E-Mails mit den Bürgern. Soziale Netzwerke wie Facebook, Microblogging-Dienste wie Twitter oder Weblogs wurden in weitaus geringerem Ausmaß für die eigene Kommunikation genutzt. Im Durchschnitt kommunizierten die Abgeordneten selbst an Informationsständen deutlich intensiver über

\begin{tabular}{|c|c|c|c|c|c|c|c|c|}
\hline & 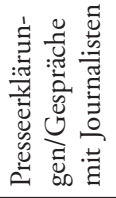 & 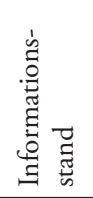 & 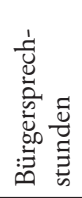 & 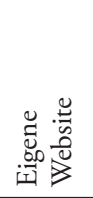 & $\begin{array}{l}0 \\
\frac{0}{0} \\
\frac{0}{20} \\
3\end{array}$ & 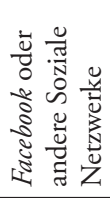 & 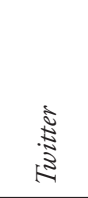 & 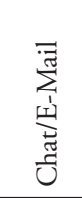 \\
\hline 2012 & $\begin{array}{c}4,23 \\
(0,75)\end{array}$ & $\begin{array}{c}3,12 \\
(1,05)\end{array}$ & $\begin{array}{c}3,86 \\
(1,00)\end{array}$ & $\begin{array}{c}4,44 \\
(0,64)\end{array}$ & $\begin{array}{c}2,00 \\
(1,19)\end{array}$ & $\begin{array}{c}3,20 \\
(1,46)\end{array}$ & $\begin{array}{c}2,25 \\
(1,57)\end{array}$ & $\begin{array}{c}4,16 \\
(0,88)\end{array}$ \\
\hline 2013 & $\begin{array}{c}4,04 \\
(0,85)\end{array}$ & $\begin{array}{c}3,17 \\
(0,91)\end{array}$ & $\begin{array}{c}3,78 \\
(0,91)\end{array}$ & $\begin{array}{c}4,15 \\
(0,80)\end{array}$ & $\begin{array}{c}1,85 \\
(1,03)\end{array}$ & $\begin{array}{c}3,60 \\
(1,30)\end{array}$ & $\begin{array}{c}2,42 \\
(1,54)\end{array}$ & $\begin{array}{c}4,07 \\
(0,94)\end{array}$ \\
\hline
\end{tabular}

Anmerkung: Antwortvorgaben jeweils von 1 („nutze ich gar nicht“) bis 5 („nutze ich sehr intensiv“). Quelle: Eigene Erhebung. 
ihre Arbeit als via Twitter oder Weblogs. Gerade bei den Sozialen Netzwerken und bei Twitter fallen allerdings die hohen Standardabweichungen auf. Dies ist ein Hinweis, dass es beim Aktivitätsgrad große Unterschiede zwischen den MdBs gibt.

Beim Zeitvergleich fällt auf, dass Twitter und vor allem Facebook 2013 stärker als Kommunikationskanal genutzt wurden als im Vorjahr. Dagegen wurde die eigene Website von den befragten Parlamentariern 2013 spürbar weniger stark genutzt als 2012.

\subsection{Eignung, Nutzung und Einfluss der Medien aus Sicht der Bundestagsabgeordneten}

Für wie geeignet halten die Abgeordneten die verschiedenen Medien und Angebote, um sich politisch zu informieren? Aus den Daten wird ersichtlich, dass Zeitungen als das am besten geeignete Medium angesehen wurden, um sich über das politische Geschehen zu informieren (vgl. Tabelle 4). Aber auch dem Fernsehen und dem Internet insgesamt wurde eine gute Eignung unterstellt. Der Blick auf einzelne Online-Angebote verdeutlicht, dass in erster Linie Online-Nachrichtenseiten und die Internetauftritte der Parteien als geeignet zur politischen Information wahrgenommen wurden. In weitaus geringerem Maße trifft

\begin{tabular}{|c|c|c|c|c|c|c|}
\hline & \multicolumn{2}{|c|}{$\begin{array}{l}\text { Wahrgenommene } \\
\text { Eignung zur politischen } \\
\text { Information* }\end{array}$} & \multicolumn{2}{|c|}{\begin{tabular}{|c|} 
Wahrgenommene \\
politische Nutzung durch \\
die Bevölkerung**
\end{tabular}} & \multicolumn{2}{|c|}{$\begin{array}{l}\text { Wahrgenommener } \\
\text { politischer Einfluss auf } \\
\text { die Bevölkerung*** }\end{array}$} \\
\hline & 2012 & 2013 & 2012 & 2013 & 2012 & 2013 \\
\hline Fernsehen & $\begin{array}{c}4,04 \\
(0,86)\end{array}$ & $\begin{array}{c}4,11 \\
(0,91)\end{array}$ & $\begin{array}{c}4,70 \\
(0,60)\end{array}$ & $\begin{array}{c}4,66 \\
(0,60)\end{array}$ & \multirow{2}{*}{$\begin{array}{c}4,40 \\
(0,71)\end{array}$} & \multirow{2}{*}{$\begin{array}{c}4,42 \\
(0,64)\end{array}$} \\
\hline Zeitungen & $\begin{array}{c}4,53 \\
(0,61)\end{array}$ & $\begin{array}{c}4,52 \\
(0,79)\end{array}$ & $\begin{array}{c}3,82 \\
(0,93)\end{array}$ & $\begin{array}{c}3,72 \\
(0,88)\end{array}$ & & \\
\hline Internet insgesamt & $\begin{array}{c}4,14 \\
(0,84)\end{array}$ & $\begin{array}{c}4,11 \\
(0,91)\end{array}$ & $\begin{array}{l}3,73 \\
(0,93)\end{array}$ & $\begin{array}{l}3,67 \\
(0,87)\end{array}$ & $\begin{array}{c}3,37 \\
(0,82)\end{array}$ & $\begin{array}{l}3,29 \\
(0,79)\end{array}$ \\
\hline $\begin{array}{l}\text { Nachrichtenseiten im } \\
\text { Internet }\end{array}$ & $\begin{array}{c}3,95 \\
(0,94)\end{array}$ & $\begin{array}{c}3,94 \\
(1,02)\end{array}$ & $\begin{array}{c}3,34 \\
(0,99)\end{array}$ & $\begin{array}{l}3,40 \\
(0,98)\end{array}$ & $\begin{array}{c}3,25 \\
(0,86)\end{array}$ & $\begin{array}{c}3,26 \\
(1,00)\end{array}$ \\
\hline $\begin{array}{l}\text { Facebook oder andere } \\
\text { Soziale Netzwerke }\end{array}$ & $\begin{array}{c}2,85 \\
(1,04)\end{array}$ & $\begin{array}{c}2,86 \\
(1,05)\end{array}$ & $\begin{array}{c}2,83 \\
(0,94)\end{array}$ & $\begin{array}{c}2,93 \\
(0,99)\end{array}$ & $\begin{array}{c}2,97 \\
(0,97)\end{array}$ & $\begin{array}{l}2,95 \\
(0,87)\end{array}$ \\
\hline Twitter & $\begin{array}{c}2,23 \\
(1,05)\end{array}$ & $\begin{array}{c}2,52 \\
(1,12)\end{array}$ & $\begin{array}{c}2,11 \\
(0,84)\end{array}$ & $\begin{array}{l}2,47 \\
(0,95)\end{array}$ & $\begin{array}{c}2,29 \\
(0,75)\end{array}$ & $\begin{array}{l}2,35 \\
(0,85)\end{array}$ \\
\hline Weblogs & $\begin{array}{l}2,43 \\
(0,85)\end{array}$ & $\begin{array}{c}2,51 \\
(0,90)\end{array}$ & $\begin{array}{c}2,02 \\
(0,73)\end{array}$ & $\begin{array}{c}2,07 \\
(0,72)\end{array}$ & $\begin{array}{l}2,08 \\
(0,67)\end{array}$ & $\begin{array}{l}2,20 \\
(0,76)\end{array}$ \\
\hline YouTube & $\begin{array}{c}2,16 \\
(0,91)\end{array}$ & $\begin{array}{c}2,25 \\
(0,87)\end{array}$ & $\begin{array}{c}2,13 \\
(0,89)\end{array}$ & $\begin{array}{l}2,23 \\
(0,96)\end{array}$ & $\begin{array}{c}2,21 \\
(0,83)\end{array}$ & $\begin{array}{c}2,24 \\
(0,81)\end{array}$ \\
\hline $\begin{array}{l}\text { Internetauftritte der } \\
\text { Parteien }\end{array}$ & $\begin{array}{c}3,42 \\
(0,97)\end{array}$ & $\begin{array}{c}3,40 \\
(1,06)\end{array}$ & $\begin{array}{c}2,40 \\
(1,01)\end{array}$ & $\begin{array}{c}2,28 \\
(0,84)\end{array}$ & $\begin{array}{c}2,64 \\
(0,75)\end{array}$ & $\begin{array}{c}2,62 \\
(0,78)\end{array}$ \\
\hline \multicolumn{7}{|c|}{$\begin{array}{l}\text { Anmerkung: Der wahrgenommene Einfluss wurde für Fernsehen und Zeitungen zusammen mit einem } \\
\text { Item erhoben. * Antwortvorgaben von } 1 \text { (,überhaupt nicht geeignet“) bis } 5 \text { (,,sehr geeignet"); ** Ant- } \\
\text { wortvorgaben von } 1 \text { (,nutzen ganz wenige Menschen") bis } 5 \text { („nutzen sehr viele Menschen“); }{ }^{* * *} \text { Ant- } \\
\text { wortvorgaben von } 1 \text { (,überhaupt kein Einfluss") bis } 5 \text { (,,sehr starker Einfluss"). } \\
\text { Quelle: Eigene Erhebung. }\end{array}$} \\
\hline
\end{tabular}


dies dagegen auf Weblogs und die Social-Media-Angebote Facebook, Twitter und YouTube zu. Dies könnte daran liegen, dass dort in erster Linie so genannter User-Generated-Content erwartet wird und weniger journalistisch-professionell aufbereiteter Inhalt. Die Eignungszuschreibungen blieben zwischen 2012 und 2013 konstant, allerdings wurde Twitter 2013 im Vergleich zum Vorjahr als spürbar geeigneter wahrgenommen. Ferner fällt auf, dass die Befunde weitgehend mit den Daten zur Mediennutzung korrespondieren: Die Parlamentarier nahmen die klassischen Massenmedien und Online-Nachrichtenseiten als geeigneter wahr und nutzten diese auch häufiger als andere Online-Angebote.

Die Abgeordneten sollten darüber hinaus einschätzen, wie viele Menschen in Deutschland die einzelnen Medien und Online-Angebote zur politischen Information nutzen. Die mit Abstand größte politische Reichweite unterstellten die Abgeordneten dem Fernsehen (vgl. Tabelle 4). Starke Nutzung vermuteten die Befragten bei Zeitungen und dem Internet; beide Medien wurden für in etwa gleich reichweitenstark gehalten. Von den einzelnen Online-Angeboten wurden den Nachrichtenseiten die meisten Nutzer zugeschrieben. Die anderen Online-Angebote wurden dagegen als weitaus weniger reichweitenstark wahrgenommen, wobei Facebook noch der vergleichsweise größte Nutzerkreis unterstellt wurde. Auch die Wahrnehmung der politischen Reichweite ist zwischen 2012 und 2013 nahezu unverändert. Eine spürbare Veränderung zeichnete sich nur bei Twitter ab, dem 2013 im politischen Kontext mehr Nutzer zugeschrieben wurden als 2012.

Die Untersuchung, für wie groß die Abgeordneten den politischen Einfluss der verschiedenen Medien und Online-Angebote auf die Bevölkerung halten (vgl. Tabelle 4), ergibt, dass den klassischen Massenmedien Fernsehen und Zeitungen eine sehr starke politische Wirkkraft unterstellt wurde - für diese deutliche Einschätzung dürfte allerdings auch ausschlaggebend sein, dass beide Medien zusammengefasst eingeschätzt werden mussten. Insbesondere Weblogs, Twitter und YouTube wurde nur geringer politischer Einfluss auf die Bevölkerung zugeschrieben. Als politisch einflussreichstes Online-Angebot sahen die Abgeordneten die Online-Nachrichtenseiten an. Die Befunde für 2012 und 2013 sind auch hier nahezu unverändert.

\subsection{Zusammenhang zwischen Nutzung und Wahrnehmung der Medien seitens der Bundestagsabgeordneten}

Wie hängt die eigene Mediennutzung mit den verschiedenen Wahrnehmungsaspekten zusammen? Plausibel ist die Annahme, dass die Abgeordneten sich vorwiegend aus solchen Medien über das politische Geschehen informieren, die sie für diesen Zweck als geeignet ansehen. Dasselbe gilt für die aktive Kommunikation. Zudem lässt sich vermuten, dass sie hierbei verstärkt solche Kanäle nutzen, denen sie eine hohe Reichweite sowie starken Einfluss auf die Bevölkerung unterstellen. Um diese Annahmen zu überprüfen, wurden mit den Daten aus dem Jahr 2013 lineare Regressionsanalysen gerechnet. Als abhängige Variable diente die Nutzungshäufigkeit der verschiedenen Medien zur Information über Politik sowie die Intensität, mit der die Abgeordneten aktiv über Facebook, Twitter und Weblogs über ihre Arbeit informieren. Die Eignung, die Reichweite sowie der politische Einfluss des jeweiligen Mediums beziehungsweise Online-Angebots auf die Bevölkerung in der Wahrnehmung der Parlamentarier bildeten die unabhängigen Variablen. Als Kontrollvariablen wurden zudem Alter, Geschlecht und Bildungsgrad (in Bildungsjahren) der MdBs berücksichtigt. 
Die Ergebnisse zeigen: Für die Häufigkeit, mit der sich die Abgeordneten über die klassischen Medien Fernsehen und Zeitungen informieren, spielen die Wahrnehmungsaspekte keine Rolle. Als signifikanter Prädiktor erweist sich hier allein das Alter: Je älter die Befragten, desto häufiger informieren sie sich über Politik aus Fernsehen und aus Zeitungen. ${ }^{21}$ Für die Social-Media Facebook, Twitter und YouTube gilt, dass sie besonders intensiv genutzt werden von jungen Parlamentariern ${ }^{22}$ und solchen Abgeordneten, die diesen Angeboten eine gute Eignung zur politischen Information unterstellen. ${ }^{23}$ Die Nutzung von Online-Nachrichtenseiten und der Webauftritte der Parteien ist unabhängig von den untersuchten Wahrnehmungsaspekten. Auch Alter, Geschlecht und Bildungsgrad haben keinen signifikanten Einfluss darauf, wie häufig sich die Abgeordneten auf diesen Wegen informieren. Für die Nutzung von Weblogs stellt lediglich das Alter einen signifikanten Prädiktor dar. ${ }^{24}$

Im Hinblick auf den Gebrauch verschiedener Online-Angebote zur Information über die eigene Arbeit zeichnet sich ein einheitliches Muster ab: Sowohl für Weblogs als Informationskanal als auch für Facebook und Twitter gilt, dass sie von jüngeren Abgeordneten intensiver genutzt werden als von älteren. ${ }^{25}$ Zudem kommunizieren die Parlamentarier intensiver über diese Kanäle, wenn sie ihnen eine gute Eignung zur politischen Information unterstellen. ${ }^{26}$ Die Wahrnehmung der Reichweite und des politischen Einflusses auf die Bevölkerung sind dagegen unerheblich für die aktive Nutzung dieser Angebote.

\section{Fazit: Vielfältiges Nutzungs- und Kommunikationsrepertoire}

Geht die gestiegene Bedeutung von Online-Medien damit einher, dass Bundestagsabgeordnete traditionelle Medien nur noch wenig nutzen und auch hergebrachte Wege der Bürgeransprache vernachlässigen? Dies lässt sich nicht bejahen. Zwar liegen keine Paneldaten vor, aus denen erkennbar wäre, ob beispielsweise ein individueller Anstieg in der Online-Nutzung im Zeitverlauf zu einem Rückgang der Zeitungslektüre führt. Allerdings sind die Zahlen auch so recht eindeutig: MdBs sind nach wie vor intensive Zeitungsleser. Ferner informieren sie sich sehr häufig durch das Fernsehen über Politik. Außerdem schreiben sie gerade den Zeitungen eine hohe Eignung zur politischen Information zu und gehen von einem sehr häufigen Fernsehkonsum in der Bevölkerung aus. Die Abgeordneten haben also die traditionellen Medien keinesfalls ausgeblendet. Dies wird nicht zuletzt an ihrem aktiven Kommunikationsverhalten erkennbar: Laut Selbstauskunft sind ihnen Presseerklärungen und Gespräche mit Journalisten weiter sehr wichtig, und zusätzlich die direkte persönliche Kommunikation mit den Bürgern.

21 Fernsehen: $\beta=0,28 ; p<.01$; Zeitungen: $\beta=0,23 ; p<.05$. Die Stichprobe der Befragung 2013 (entstanden aus einer Vollerhebung) ist zumindest mit Blick auf die Fraktionszugehörigkeit kein repräsentatives Abbild der Grundgesamtheit. Daher sind Rückschlüsse auf diese Grundgesamtheit mit Vorsicht zu behandeln. Vertiefende Analysen ergaben allerdings keine systematischen Einflüsse der Fraktionszugehörigkeit auf die Nutzung von Medien und die aktive Kommunikation.

22 Facebook: $\beta=-0,25 ; p<.01$; Twitter: $\beta=-0,36 ; p<.001$; YouTube: $\beta=-0,20 ; p<.05$.

23 Facebook: $\beta=0,39 ; p<.001$; Twitter: $\beta=0,42 ; p<.001$; YouTube: $\beta=0,24 ; p<.05$.

24 Weblogs: $\beta=-0,25 ; p<.01$.

25 Weblogs: $\beta=-0,18 ; p<.05$; Facebook: $\beta=-0,24 ; p<.01$; Twitter: $\beta=-0,30 ; p<.001$.

26 Weblogs: $\beta=0,22 ; p<.05$; Facebook: $\beta=0,32 ; p<.01$; Twitter: $\beta=0,46 ; p<.001$. 
Gleichzeitig belegen die Befunde den hohen Stellenwert, den die Parlamentarier den OnlineMedien zuweisen. Sie nutzen das Internet intensiv - um sich selbst zu informieren, aber auch, um über ihre Arbeit zu kommunizieren. Gerade den Online-Nachrichtenseiten sprechen sie eine hohe Eignung zur politischen Information zu und nehmen an, dass diese sehr häufig von der Bevölkerung gelesen werden. Wie in anderen Studien zeigt sich auch, dass vor allem jüngere Abgeordnete stärker von Online-Medien Gebrauch machen. Keineswegs kann jedoch aus den Ergebnissen gefolgert werden, dass die älteren Abgeordneten sich den Online-Medien verweigern. Außerdem ist gerade bei den Social-Media-Angeboten die wahrgenommene Eignung wichtig: Die MdBs schätzen deren Eignung zur politischen Information unterschiedlich ein - je stärker sie überzeugt sind, desto eher nutzen sie diese Medien.

Die Befunde sind mit Blick auf die beiden Erhebungszeiträume sehr stabil. Eine Tendenz ist jedoch unverkennbar: Der Stellenwert der Kommunikation über die eigene Website sinkt. Die aktive Kommunikation über Twitter und insbesondere über Facebook nimmt dagegen zu, ebenso der Gebrauch von Facebook oder anderen Sozialen Netzwerken zur politischen Information. Die Anstiege zwischen den Befragungen 2012 und 2013 sind deutlich. Der Vernetzungsgedanke wird also offenbar wichtiger. Ein Grund hierfür kann darin liegen, dass sich die Abgeordneten versprechen, über Soziale Medien eine größere Zahl an Adressaten zu erreichen - laut ihrer Einschätzung nutzen zum Bespiel weitaus mehr Menschen Facebook als die Internetauftritte der Parteien, um sich politisch zu informieren. Zudem könnte die Hoffnung darin bestehen, dass über Social-Media-Kanäle verbreitete Inhalte auch von den klassischen Massenmedien aufgegriffen werden - etwa indem eine über Twitter vermittelte Stellungnahme Eingang in Zeitungsartikeln findet.

Insgesamt machen diese Ergebnisse zunächst deutlich, dass sich das Nutzungs- und Kommunikationsrepertoire der Abgeordneten des Bundestags erweitert hat. Die neuen Optionen der Online-Welt werden parallel zu den traditionellen Möglichkeiten der Nutzung und Kommunikation berücksichtigt, und zwar sowohl als Quelle für politische Informationen als auch zur Ansprache von Bürgern und anderen Gruppen. Dieses Nutzungsverhalten ist durchaus rational und keineswegs widersprüchlich: Einerseits versuchen die Parlamentarier auf diese Weise, Erwartungen an moderne Kommunikation im Zeitalter von Online-Medien zu erfüllen. Andererseits verlieren sie die nach wie vor einflussreiche traditionelle Medienwelt nicht aus den Augen. Darüber hinaus erwiesen sie sich als lernfähig: Sie haben ganz offensichtlich erkannt, dass sich aus einer komplementären Nutzung alter und neuer Medien die Chance ergibt, mit größerer Wahrscheinlichkeit relevante Informationen zu erhalten denn schließlich werden beispielsweise auch über soziale Netzwerke Nachrichten aus klassischen Massenmedien verbreitet - und umgekehrt unterschiedliche Zielgruppen vielfältig mit eigenen Informationen zu bedienen. Dies gilt grundsätzlich für alle Abgeordneten. Auch wenn jüngere online-affiner sind, haben sie nicht als einzige ihr Medienrepertoire ergänzt.

Die Befunde sind allerdings deshalb bemerkenswert, da ein komplementärer Gebrauch von On- und Offlinemedien mit hohen Anforderungen verbunden ist: Abgesehen von dem vermehrten zeitlichen Aufwand steigert diese zweigleisige Strategie die Komplexität des Kommunikationsverhaltens enorm. Die Parlamentarier müssen nicht länger nur die Logiken traditioneller Medien beachten, sondern auch die der Online-Welt. Dies bedeutet zum Beispiel, sich entsprechende technische Kompetenzen anzueignen oder Personal zu beschäftigen, um die Social-Media-Profile beständig zu aktualisieren, Bürgeranfragen per E-Mail zu beantworten oder um die eigene Website kontinuierlich mit aktuellen Videos, Fotostrecken oder Informationen zu pflegen. Auch sollten Online- und Offline-Kommunikations- 
wege stets aufeinander abgestimmt werden, damit ein einheitliches Bild der eigenen Arbeit vermittelt werden kann.

Aus den vorgestellten Befunden lassen sich Konsequenzen ableiten. Zum einen zeigt sich, dass es auch in Zukunft notwendig sein wird, klassische Medien nicht aus den Augen zu verlieren. Selbst wenn diesen häufig, wie im Falle der Zeitungen, eine problematische Zukunft prognostiziert wird, haben sie in dem spezifischen Elitesegment der Bundestagsabgeordneten eine treue Leserschaft. Studien, die ausschließlich auf die Online-Kommunikation von Politikern gerichtet sind, vernachlässigen somit einen großen, vielleicht sogar den (noch) überwiegenden Teil ihrer Informationsquellen und Kommunikationsaktivitäten. Gleiches gilt für PR-Aktivitäten und ähnliche Versuche der Ansprache von Abgeordneten, die ausschließlich über Online-Medien erfolgen.

Zum anderen dürfte es hilfreich sein, nicht nur die Mediennutzung an sich zu erfassen, sondern auch die Wahrnehmung von Medien. In dieser Hinsicht lassen die vorliegenden Befunde einerseits eine gewisse Passung zwischen Nutzung und Wahrnehmung erkennen: Medien, denen eine gute Eignung zur politischen Information unterstellt wird, werden häufig auch stärker genutzt. Andererseits greifen die Parlamentarier auf Online-Angebote teilweise durchaus intensiv zurück, obwohl sie ihnen eine eher geringe Reichweite und einen schwachen politischen Einfluss auf die Bevölkerung unterstellen. Dies könnte ein Hinweis darauf sein, dass die aktive Nutzung von Online-Medien keiner ausschließlichen Kosten-Nutzen-Rechnung folgt, sondern auch andere Motive eine Rolle spielen. Zu denken wäre hier etwa schlicht an die Sorge, ohne eigene Online-Aktivitäten hinter andere Abgeordnete zurückzufallen und als nicht zeitgemäß wahrgenommen zu werden. Dieses Ergebnis deutet jedoch auch darauf hin, dass möglicherweise weniger die allgemeine Bevölkerung direkt im Fokus der Social-Media-Aktivitäten der Abgeordneten steht, sondern vielmehr spezifische Gruppen angesprochen werden sollen. Neben dem eigenen Unterstützerkreis könnten dies Meinungsführer (zum Beispiel Journalisten oder andere Politiker) sein - verbunden mit der Hoffnung, dass letztere im Sinne des Mehrstufenflusses der Kommunikation $^{27}$ die Informationen an größere Bevölkerungsgruppen verbreiten.

Zusätzliche Konsequenzen für weitere Forschung ergeben sich aus den Beschränkungen der vorgestellten Studien: Zwar wurden zwei Befragungen durchgeführt; ihr zeitlicher Abstand war jedoch zu gering, um langfristige Tendenzen zu erfassen. Weitere Befragungen zu späteren Zeitpunkten sind daher notwendig. Ferner wurden neben den Wahrnehmungen ausschließlich Nutzungs- und Kommunikationsintensitäten gemessen; dabei wurde zwischen verschiedenen Online-Medien differenziert, Zeitungen und Fernsehen wurden dagegen nur pauschal erfasst. Die MdBs machten zudem selbst Angaben; eine objektive Erfassung der tatsächlichen Nutzung und der tatsächlichen Aktivitäten ist nicht erfolgt. Gerade dies könnte Hinweise darauf erbringen, ob die Abgeordneten beispielsweise einzelne Kanäle mit exklusiven Informationen versorgen oder ob sie gleiche Inhalte auf sämtlichen ihnen zur Verfügung stehenden Wegen vermitteln. Dies könnte mit Selbstauskünften der Abgeordneten verknüpft werden, aus welchen Gründen sie welche Kanäle für die politische Information und für ihre eigene Kommunikation nutzen. Auf diese Weise würde ein vollständigeres Bild entstehen, inwiefern sich On- und Offline-Medien in ihren Funktionen ergänzen beziehungsweise voneinander abgrenzen lassen.

27 Vgl. Paul F. Lazarsfeld / Bernard Berelson / Hazel Gaudet, The People’s Choice. How the Voter Makes Up His Mind in a Presidential Campaign, New York 1944. 